\title{
PREVALENCIA Y CARACTERIZACIÓN DE GESTANTES SEROPOSITIVAS PARA SARS-CoV-2
}

\author{
Enrique Guevara-Ríos ${ }^{1,2, a, b}$, Cesar Carranza-Asmat ${ }^{1,2, a, b}$, Kelly Zevallos-Espinoza ${ }^{1, a}$, Marcos Espinola-Sánchez ${ }^{1, c}$, \\ Pedro Arango-Ochante ${ }^{1, a}$, Félix Dasio Ayala-Peralta ${ }^{1,2, a, b}$, Ricardo Álvarez-Carrasco ${ }^{1, d}$, Antonio Luna-Figueroa ${ }^{1,2, a, b}$, \\ Luis Meza-Santibañez ${ }^{1, a}$, Carlos Pérez-Aliaga ${ }^{1, a}$, Augusto Racchumí-Vela ${ }^{1, \mathrm{e}}$.
}

\begin{abstract}
RESUMEN
Objetivo: Describir la prevalencia y características de las gestantes seropositivas para SARS-CoV-2. Materiales y métodos: Estudio cuantitativo, transversal y descriptivo. Realizado en gestantes tamizadas para SARS-CoV-2 que cumplieron criterios de selección al ingreso hospitalario. Se recogieron variables de las características maternas, complicaciones del embarazo y condición del recién nacido a partir del historial médico respectivo. El análisis de datos se realizó utilizando estadígrafos descriptivos e intervalos de confianza al 95\%. Resultados: Se tamizaron 1477 embarazadas ingresadas a hospitalización, identificando una seroprevalencia de anticuerpos anti-SARS-CoV-2 en el 5.28\% (IC95\%: 4.22\%-6.57\%). Entre los casos de seroprevalencia positiva, se observó lgM $11.5 \%$ (IC95\%: 5.7\%-21.3\%), IgM/IgG 64.1\% (IC95\%: 5.7\%-21.3\%), IgG 19\% (IC95\%: 15.7\% y 35.6\%), 91\% de gestantes seropositivas fueron asintomáticas, $40 \%$ de gestantes seropositivas presentó algún tipo de complicación del embarazo, siendo más frecuente ruptura prematura de membranas $(14.1 \%)$, aborto $(4.1 \%)$ y amenaza de parto pretermino $(4.1 \%)$. De las gestantes que culminaron en parto el $69.6 \%$ fue vía vaginal, encontrándose un $11.6 \%$ de parto pretermino. No hubo muertes maternas ni muertes en recién nacidos. Conclusión: Las gestantes con criterios de hospitalización para atención del parto o por morbilidad del embarazo presentan una prevalencia de serología positiva para SARS-CoV-2 del 5.28\%; mayormente son asintomáticas. No se descarta posibles efectos del virus SARS-CoV-2 ante los hallazgos de parto pretermino, ruptura prematura de membrana y aborto espontaneo.
\end{abstract}

Palabras Clave: COVID-19; Infección por Coronavirus; Anticuerpos; Gestación; Complicaciones del embarazo (Fuente: DeCS BIREME).

\section{PREVALENCE AND CHARACTERIZATION OF SEROPOSITIVE PREGNANT FOR SARS-COV-2}

\begin{abstract}
Objective: To describe the prevalence and characteristics of seropositive pregnant women for SARS-CoV-2. Materials and methods: Quantitative, cross-sectional and descriptive study. Performed in pregnant women screened for SARS-CoV-2 who met selection criteria at hospital admission. Variables of maternal characteristics, pregnancy complications and newborn condition were collected from the respective medical history. Data analysis was performed using descriptive statistics and $95 \%$ confidence intervals. Results: 1477 pregnant women admitted to hospitalization were screened, identifying a seroprevalence of anti-SARSCoV-2 antibodies in 5.28\% (95\% Cl: $4.22 \%-6.57 \%)$. Among the cases of positive seroprevalence, IgM $11.5 \%(95 \% \mathrm{Cl}: 5.7 \%$ $-21.3 \%$ ), IgM / IgG 64.1\% (95\% Cl: $5.7 \%-21.3 \%$ ), IgG 19\% (95\% Cl: $15.7 \%$ and 35.6 ) were observed $\%$ ), $91 \%$ of seropositive pregnant women were asymptomatic, $40 \%$ of seropositive pregnant women presented some type of pregnancy complication, being more frequent premature rupture of membranes $(14.1 \%)$, abortion $(4.1 \%)$ and threat of preterm delivery $(4.1 \%)$. Of the pregnant women who finished in labor, $69.6 \%$ were vaginally, and $11.6 \%$ were preterm. There were no maternal deaths or newborn deaths. Conclusion. Pregnant women with hospitalization criteria for delivery care or pregnancy morbidity have a prevalence of positive serology for SARS-CoV-2 of 5.28\%; they are mostly asymptomatic. Possible effects of the SARS-CoV-2 virus are not ruled out due to the findings of preterm labor, premature membrane rupture and spontaneous abortion.
\end{abstract}

Key Words: COVID-19; Coronavirus infection; Antibodies; Pregnancy; Pregnancy complications (Source: MeSH NLM).

\section{INTRODUCCIÓN}

En diciembre del 2019 se notificaron casos de infección por el nuevo coronavirus, cuyo brote se originó por exposición a un mercado de pescados y mariscos de Huanan, en la ciudad de Wuhan, China'. Se conoce actualmente que el SARS-Cov-2 es el causante de este brote, que se clasifica dentro del género de la subfamilia Orthocoronavirinae dentro de la familia Coronaviridae, y muestra una fuerte afinidad por los receptores respiratorios humanos ${ }^{2}$.

El 31 de enero la OMS declaró, lo que hasta ese momento era una epidemia, Emergencia de Salud Pública de Preocupación Internacional, con 9826 casos nuevos, la mayoría en China y el resto de casos en otros países asiáticos, Europa y América del Norte (USA y Canadá) para

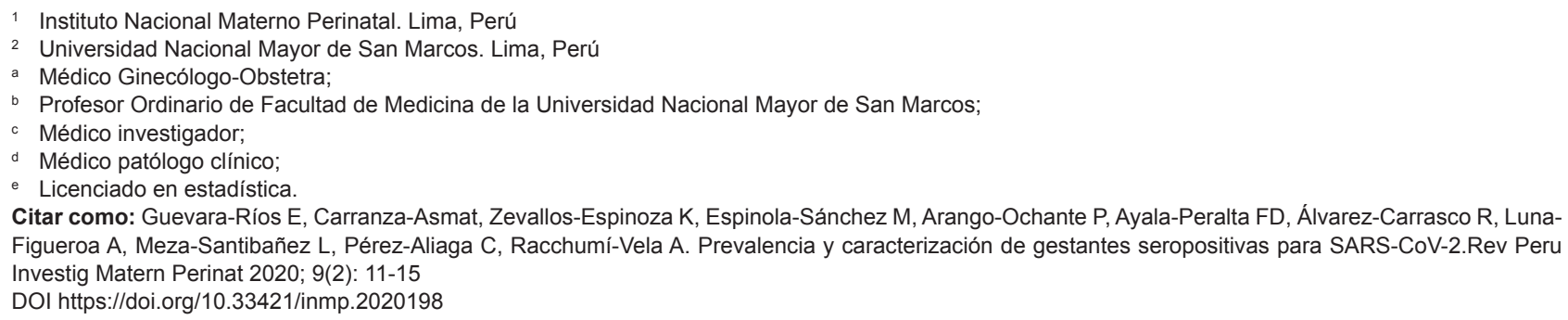

Recibido: 13-06-20 Aprobado: 30-06-20 
esa fecha ${ }^{3}$. Al 4 de junio de este año, tenemos 6'416,828 casos confirmados y 382,867 pacientes fallecidos a causa del COVID-19 a nivel mundial ${ }^{4}$.

El primer caso en nuestro país fue notificado al Centro Nacional de Epidemiologia, Prevención y Control de Enfermedades del Ministerio de Salud el 5 de marzo del presente año. El paciente tuvo viajes previos a Madrid, Paris, Republica Checa y Barcelona, llegando finalmente a Lima vía aérea el 26 de febrero. Posterior a la confirmación del caso se realiza una búsqueda activa de contactos ${ }^{5}$.Sin embargo, los casos fueron en aumento y el gobierno peruano tuvo que tomar medidas drásticas de aislamiento social entre otras. A pesar de las medidas tomadas el número de casos asciende a 183,198 casos positivos confirmados con pruebas rápidas y moleculares y 5,031 personas fallecidas a causa del COVID-19 para un corte realizado al 4 de junio ${ }^{6}$.

El 22 de febrero de 2020 se notificó un caso probable de transmisión vertical en el Hospital Renmin, Wuhan, China. El recién nacido de madre con diagnóstico de COVID-19 tuvo prueba serológica positiva en valores elevados para IgM e lgG, de muestra tomada en el post parto inmediato, teniendo en cuenta que los anticuerpos tipo IgM no pasan a través de la placenta ${ }^{7,8}$. Muchos otros estudios han reportado datos similares, sin embargo existe controversia de resultados serológicos y moleculares para la definición de Transmisión Neonatal, porque los resultados de pruebas moleculares para estos neonatos fueron negativos.

El Instituto Nacional Materno Perinatal no es ajeno a esta situación. La institución recibe gestantes con diagnóstico clínico y serológico de COVID-19 y también se ha observado un número importante de neonatos infectados por esta enfermedad. Sabemos también que las gestantes son particularmente susceptibles a los patógenos respiratorios y a la neumonía grave, debido a los cambios fisiológicos en los sistemas inmunitario y cardiopulmonar (p.ej., elevación del diafragma, aumento del consumo de oxígeno y edema de la mucosa del tracto respiratorio), que pueden volverlos intolerantes a la hipoxia y mayor complicación por las mismas ${ }^{9}$.

El objetivo de esta investigación es describir la prevalencia y características de las gestantes seropositivas para SARS-CoV-2.

\section{MATERIALES Y MÉTODOS}

Estudio cuantitativo, observacional y descriptivo.

En el estudio participaron mujeres embarazadas atendidas en el Instituto Nacional Materno Perinatal de Lima, Perú, entre el 15 de abril y 01 de mayo del 2020, que cumplieron los siguientes criterios de inclusión: a) gestante con ingreso a hospitalización; c) gestante con resultado de prueba serológica anti-SARS-CoV-2 al momento del ingreso hospitalario. Criterios de exclusión: a) historial médico incompleto para las variables de estudio.
El estudio fue realizado en Instituto Nacional Materno Perinatal del Ministerio de Salud del Perú, un establecimiento del tercer nivel de atención, con categoría III-2. Debido a la declaratoria del estado de emergencia sanitaria a nivel nacional por la pandemia de COVID-19, en el periodo de estudio se realizaron atenciones en el servicio de emergencia donde efectúo pruebas serológicas de anticuerpos anti-SARS-CoV-2 a toda gestante que tenga criterios de hospitalización, indicado por el médico especialista, para la atención del parto o por morbilidad en el embarazo.

Prueba de detección de anticuerpos anti-SARS-CoV-2: La prueba utilizada en la institución fue el One Step Test Kit Covid-19, prueba rápida que cuenta con la certificación y autorización de la Comunidad Europea.

La prueba empleada cuenta con la certificación internacional ISO 9001 e ISO 13485 (Inserto de la prueba One Step Test Kit Covid-19. Core Technology Company Limited de Beijing, China), siendo la muestra utilizada el suero/ plasma, y el método de inmunocromatografía para detectar simultáneamente las inmunoglobulinas $\lg \mathrm{M}$ e $\lg \mathrm{G}$.

Para este procedimiento e interpretación de resultados, se siguieron las indicaciones del inserto de la prueba (One Step Test Kit Covid-19): Luego de 15 minutos de ejecutado la prueba de inmunocromatografia se procedió a la lectura en el dispositivo casete. Se consideró un resultado positivo cuando se observó una línea roja en el área T1 (inmunoglobulina Ig M) y/o en el área T2 (inmunoglobulina Ig G); y se consideró Negativa cuando no apareció la línea roja en ambas áreas, T1 y T2. El ensayo quedó validado cuando se observó una línea roja en el área de control, de lo contrario se repitió la prueba, porque no se habían cumplido los parámetros establecidos por el fabricante.

Los resultados de la prueba fueron registrados en una ficha estándar de pruebas serológicas de la oficina de Epidemiologia y Salud Ambiental del Instituto Nacional Materno Perinatal.

Variables: Se estudiaron la edad materna indicada en años cumplidos previo al embarazo y clasificada en edad adolescente (menor a 19 años), edad materna adulta (de 19 a 34 años) y edad materna avanzada (de 35 años a más); trimestre de gestación según fecha de ultima regla o ecografía del primer trimestre; paridad; resultado de prueba rápida, indicado como $\operatorname{lgM}$, IgG o ambas; controles prenatales considerado como control adecuado al número de controles mayor o igual a 6 registrados; nivel educativo; estado civil; síntomas respiratorios presentes o ausentes (correspondientes a tos, dolor de garganta, cefalea, fiebre, escalofrió y/o congestión nasal) indicados al ingreso hospitalario; tipo de complicación en el embarazo.

Recolección de la información: Los resultados de la prueba rápida aplicada a cada gestante, durante el 
periodo de estudio, fueron obtenidos a partir de las fichas estandarizadas de pruebas serológicas de la oficina de Epidemiologia y Salud Ambiental del Instituto Nacional Materno Perinatal; la recolección de información respecto a las variables del estudio como características de la madre, síntomas respiratorios al ingreso de hospitalización y complicaciones del embarazo fueron obtenidas a partir de la historia clínica de la gestante. Se protegió la identidad de las participantes mediante codificación, garantizando la protección de la identidad y datos respectivos.

Análisis estadístico: El análisis inició con el pre procesamiento de datos, el análisis exploratorio de los datos obtenidos, y para la descripción de los resultados se utilizó estadística descriptiva mediante tablas de contingencia con resúmenes de frecuencias absolutas y relativas. El análisis inferencial consistió en la estimación de intervalos de confianza al 95\%. El procesamiento de los datos en el software estadístico R versión 4.0.

Consideraciones éticas: La investigación tuvo la aprobación del Comité de Ética en Investigación, del Instituto Nacional Materno Perinatal, y el permiso institucional correspondiente.

\section{RESULTADOS}

En el periodo de estudio se evaluaron 1488 embarazadas, de las cuales 1477 cumplieron con los criterios de selección establecidos en el estudio, de ellas 78 tuvieron anticuerpos
Tabla 1. Distribución de los resultados de prueba serológica en gestantes tamizadas. Instituto Nacional Materno Perinatal. Abril, 2020.

\begin{tabular}{lcc}
\hline $\begin{array}{l}\text { Resultado de } \\
\text { prueba serológica }\end{array}$ & $\mathrm{N}=1477$ & $\begin{array}{l}\text { Porcentaje } \\
\text { (IC al 95\%) }\end{array}$ \\
\hline Positiva & 78 & $5.28 \%(4.2 \%-6.6 \%)$ \\
Negativa & 1399 & $94.7 \%(93.4 \%-95.8 \%)$ \\
$\begin{array}{l}\text { Tipo de serología } \\
\text { positiva }\end{array}$ & $\mathrm{n}=78$ & Porcentaje (IC al 95\%) \\
IgM & 9 & $11.5 \%(5.7 \%-21.3 \%)$ \\
IgM/IgG & 50 & $64.1 \%(52.4 \%-74.4 \%)$ \\
IgG & 19 & $24.4 \%(15.7 \%-35.6 \%)$ \\
\hline
\end{tabular}

Fuente: Elaboración propia.

anti-SARS-CoV-2, lo que significó una seroprevalencia de 5.28\% (IC95\%: 4.22\%-6.57\%) en embarazadas tamizadas ingresadas a hospitalización. Entre los casos de seroprevalencia positiva, se observó IgM 11.5\% (IC95\%: $5.7 \%-21.3 \%$ ), IgM/lgG 64.1\% (IC95\%: 5.7\%-21.3\%) e IgG 19\% (IC95\%: $15.7 \%$ y $35.6 \%$ ) (Tabla 1 ).

En el grupo de gestantes que dieron resultado positivo a la prueba rápida, se observó que el $70.5 \%$ fueron mujeres con edad entre 19 a 34 años, $28.2 \%$ en edad mayor a 35 años y solo un $1.3 \%$ en edad menor a 19 años; siendo la serología más frecuente de tipo lgM/lgG en todos los grupos etarios. Se observó con mayor frecuencia gestantes atendidas durante en el tercer trimestre de gestación, control prenatal no adecuada, multíparas, conviviente/casada, con nivel de educación de secundaria y como ocupación ama de casa; siendo la serología de anticuerpo más frecuente de tipo lgM/lgG anti-SARS-CoV-2 (Tabla 2).

Tabla 2. Caracterización de gestantes según respuesta de anticuerpos anti-SARS-CoV-2 en gestantes. Instituto Nacional Materno Perinatal. Abril, 2020.

\begin{tabular}{|c|c|c|c|c|c|c|c|c|}
\hline \multirow{2}{*}{ Características } & \multicolumn{2}{|c|}{ Total } & \multicolumn{2}{|c|}{$\lg G$} & \multicolumn{2}{|c|}{$\lg M$} & \multicolumn{2}{|c|}{ lgG/lgM } \\
\hline & $\mathbf{n}$ & $\%$ & $\mathbf{n}$ & $\%$ & $\mathbf{n}$ & $\%$ & $n$ & $\%$ \\
\hline \multicolumn{9}{|l|}{ Edad materna } \\
\hline Adolescentes & 1 & $1.3 \%$ & 1 & $5.3 \%$ & 0 & $0.0 \%$ & 0 & $0.0 \%$ \\
\hline Adulta & 55 & $70.5 \%$ & 13 & $68.4 \%$ & 7 & $77.8 \%$ & 35 & $70.0 \%$ \\
\hline $\begin{array}{l}\text { Edad materna avanzada } \\
\text { Edad gestacional }\end{array}$ & 22 & $28.2 \%$ & 5 & $26.3 \%$ & 2 & $22.2 \%$ & 15 & $30.0 \%$ \\
\hline Primer trimestre & 6 & $7.7 \%$ & 3 & $15.8 \%$ & 2 & $22.2 \%$ & 1 & $2.0 \%$ \\
\hline Segundo trimestre & 1 & $1.3 \%$ & 0 & $0.0 \%$ & 0 & $0.0 \%$ & 1 & $2.0 \%$ \\
\hline Tercer trimestre & 71 & $91.0 \%$ & 16 & $84.2 \%$ & 7 & $77.8 \%$ & 48 & $96.0 \%$ \\
\hline \multicolumn{9}{|l|}{ Paridad } \\
\hline Nulípara & 17 & $21.8 \%$ & 3 & $15.8 \%$ & 2 & $22.2 \%$ & 12 & $24.0 \%$ \\
\hline Primípara & 28 & $35.9 \%$ & 7 & $36.8 \%$ & 3 & $33.3 \%$ & 18 & $36.0 \%$ \\
\hline Multípara & 33 & $42.3 \%$ & 9 & $47.4 \%$ & 4 & $44.4 \%$ & 20 & $40.0 \%$ \\
\hline \multicolumn{9}{|l|}{ Estado civil } \\
\hline Casado & 5 & $6.4 \%$ & 2 & $10.5 \%$ & 0 & $0.0 \%$ & 3 & $6.0 \%$ \\
\hline Conviviente & 64 & $82.1 \%$ & 14 & $73.7 \%$ & 8 & $88.9 \%$ & 42 & $84.0 \%$ \\
\hline Soltero & 9 & $11.5 \%$ & 3 & $15.8 \%$ & 1 & $11.1 \%$ & 5 & $10.0 \%$ \\
\hline Nivel de educación & 0 & & & & & & & \\
\hline Primaria & 4 & $5.1 \%$ & 0 & $0.0 \%$ & 1 & $11.1 \%$ & 3 & $6.0 \%$ \\
\hline Secundaria & 62 & $79.5 \%$ & 16 & $84.2 \%$ & 7 & $77.8 \%$ & 39 & $78.0 \%$ \\
\hline Superior & 12 & $15.4 \%$ & 3 & $15.8 \%$ & 1 & $11.1 \%$ & 8 & $16.0 \%$ \\
\hline \multicolumn{9}{|l|}{ Ocupación } \\
\hline Ama de casa & 68 & $87.2 \%$ & 15 & $78.9 \%$ & 9 & $100.0 \%$ & 44 & $88.0 \%$ \\
\hline Independiente & 7 & $9.0 \%$ & 3 & $15.8 \%$ & 0 & $0.0 \%$ & 4 & $8.0 \%$ \\
\hline Empleado & 2 & $2.6 \%$ & 0 & $0.0 \%$ & 0 & $0.0 \%$ & 2 & $4.0 \%$ \\
\hline Estudiante & 1 & $1.3 \%$ & 1 & $5.3 \%$ & 0 & $0.0 \%$ & 0 & $0.0 \%$ \\
\hline
\end{tabular}

Fuente: Elaboración propia. 
Se encontró que el $91 \%$ de gestantes con anticuerpos antiSARS-CoV-2 fue asintomático, y la presencia de síntomas fue más frecuente en gestantes con anticuerpos IgM antiSARS-CoV-2. El tipo de anticuerpo más frecuentemente identificado en gestantes con complicaciones en el embarazo fue $\mathrm{lgM} / \mathrm{lgG}$. De las pacientes con anticuerpos anti-SARS-CoV-2, el $39.7 \%$ presento algún tipo de complicación en el embarazo, siendo más frecuente ruptura prematura de membranas, aborto, amenaza de parto pretermino y preeclampsia (Tabla 3 ).

Tabla 3. Descripción de presencia de síntomas respiratorios y complicaciones del embarazo según respuesta de anticuerpos anti-SARS-CoV-2 en gestantes. Instituto Nacional Materno Perinatal. Abril, 2020

\begin{tabular}{ccccccccc}
\hline & \multicolumn{2}{c}{ Total } & \multicolumn{2}{c}{ IgG } & \multicolumn{2}{c}{ IgM } & \multicolumn{2}{c}{ IgG/lgM } \\
\cline { 2 - 9 } & $\mathbf{n}$ & $\%$ & $\mathbf{n}$ & $\%$ & $\mathbf{n}$ & $\%$ & $\mathbf{n}$ & $\%$ \\
\hline Síntomas respiratorios & & & & & & \\
No & 71 & $91.0 \%$ & 17 & $89.5 \%$ & 7 & $77.8 \%$ & 47 & $94.0 \%$ \\
Si & 7 & $9.0 \%$ & 2 & $10.5 \%$ & 2 & $22.2 \%$ & 3 & $6.0 \%$
\end{tabular}

\section{Complicaciones en el embarazo}

\begin{tabular}{|c|c|c|c|c|c|c|c|c|}
\hline Aborto & 4 & $5.1 \%$ & 2 & $10.5 \%$ & 1 & $11.1 \%$ & 1 & $2.0 \%$ \\
\hline $\begin{array}{l}\text { Parto } \\
\text { pretérmino }\end{array}$ & 8 & $11.6 \%$ & 2 & $12.5 \%$ & 1 & $16.7 \%$ & 5 & $10.6 \%$ \\
\hline $\begin{array}{l}\text { Embarazo } \\
\text { ectópico }\end{array}$ & 1 & $1.3 \%$ & 1 & $5.3 \%$ & 0 & $0.0 \%$ & 0 & $0.0 \%$ \\
\hline $\begin{array}{l}\text { Hemorragia de } \\
\text { segunda mitad } \\
\text { del embarazo }\end{array}$ & 1 & $1.3 \%$ & 0 & $0.0 \%$ & 0 & $0.0 \%$ & 1 & $2.0 \%$ \\
\hline $\begin{array}{l}\text { Hiperemesis } \\
\text { gravídica }\end{array}$ & 1 & $1.3 \%$ & 0 & $0.0 \%$ & 1 & $11.1 \%$ & 0 & $0.0 \%$ \\
\hline $\begin{array}{l}\text { Insuficiencia } \\
\text { placentaria }\end{array}$ & 2 & $2.6 \%$ & 1 & $5.3 \%$ & 0 & $0.0 \%$ & 1 & $2.0 \%$ \\
\hline Preeclampsia & 4 & $5.1 \%$ & 1 & $5.3 \%$ & 0 & $0.0 \%$ & 3 & $6.0 \%$ \\
\hline $\begin{array}{l}\text { Ruptura } \\
\text { prematura de } \\
\text { membranas }\end{array}$ & 11 & $14.1 \%$ & 2 & $5.3 \%$ & 0 & $0.0 \%$ & 9 & $16.0 \%$ \\
\hline $\begin{array}{l}\text { Trabajo } \\
\text { de parto } \\
\text { disfuncional }\end{array}$ & 1 & $1.3 \%$ & 0 & $0.0 \%$ & 0 & $0.0 \%$ & 1 & $0.0 \%$ \\
\hline $\begin{array}{l}\text { Infección } \\
\text { urinaria }\end{array}$ & 1 & $1.3 \%$ & 0 & $0.0 \%$ & 0 & $0.0 \%$ & 1 & $2.0 \%$ \\
\hline
\end{tabular}

Fuente: Elaboración propia.

\section{DISCUSIÓN}

El embarazo representaría una condición de probable riesgo en el contexto de la actual pandemia por el nuevo coronavirus. Esto debido a los cambios hormonales y la estructura fisiológica, el sistema respiratorio experimenta grandes cambios como el incremento de la ventilación pulmonar y el consumo de oxígeno. El aumento de los niveles de estrógenos durante el embarazo puede causar congestión y secreción excesiva de células epiteliales del tracto respiratorio, que lo hace propenso a infecciones y obstrucciones del tracto respiratorio ${ }^{10}$. Sin embargo, en el estudio se observó que las gestantes son principalmente asintomáticas, y los casos seropositivos presentaban un clínica leve.
En este estudio observamos una prevalencia del $5.28 \%$ de gestantes seropositivas para SARS COV-2, entre las gestantes ingresadas a hospitalización. El 11.5\% fue $\mathrm{IgM}$ positivo, $64.1 \% \mathrm{lgM} / \mathrm{lgG}$ positivo y el $19 \%$ IgG positivo, observamos que la mayor cantidad de pacientes acuden al hospital en la etapa subaguda del covid-19 y el motivo de su ingreso fue principalmente por problemas obstétricos. Con respecto a las características epidemiológicas se mantiene el perfil epidemiológico de años anteriores en el Instituto Nacional Materno Perinatal; respecto al perfil obstétrico, una diferencia encontrada en el presente estudio es la paridad, ya que años anteriores el grupo predominante fue el de nulíparas con un $41 \%$ en promedio ${ }^{11}$, mientras que en el estudio tenemos a las multíparas con el $42 \%$.

Con respecto a los síntomas, las pacientes asintomáticas respiratorias al momento del ingreso a hospitalización representaron el $91 \%$ similar a lo reportado por otros autores $^{12,13}$ esto difiere con un estudio poblacional en base al registro del sistema de vigilancia obstétrica del reino unido donde al menos el $65 \%$ de las pacientes presentaron síntomas ${ }^{14}$. En base a lo encontrado, se considera que el manejo e interrupción del embarazo debe basarse en la condición obstétrica y la seguridad de la madre, más que en el diagnóstico de COVID-19; ya que los casos severos son escasos en la mayoría de las series, debido a que es una intercurrencia que estamos conociendo, esta deberá ser monitorizada cercanamente.

La morbilidad es mayor en las pacientes con lgG/lgM, dentro de las patologías principales se encuentran la ruptura prematura de membranas con un $14 \%$, parto pretérmino con $12 \%$ y aborto con $5,1 \%$; los casos identificados es superior al histórico observado en las atenciones en emergencia de nuestra institución, que corresponde al $5,5 \%, 3 \%$ y $2,8 \%$ respectivamente ${ }^{11}$. No ocurre lo mismo con la preeclampsia que el histórico tiene como promedio $7 \%$ pero nosotros encontramos el $5,1 \%$, contrario a otros estudios donde la prevalencia fue mayor $^{5,15}$. Estas diferencias observadas pueden estar sujetas a la temporalidad del estudio y de la muestra analizada.

El presente estudio tiene limitaciones al ser un estudio retrospectivo y en el contexto de la pandemia de COVID-19 que acontece en una población como la nuestra. Los datos presentados se recopilaron posteriormente a la atención en un periodo de 15 días y es probable que la dinámica cambie o tenga una tendencia errática, una vez que estén disponibles un mayor conjunto de datos. No se ha encontrado ningún caso de mortalidad materna relacionada por COVID-19 en el presente estudio.

En conclusión, las gestantes con criterios de hospitalización para atención del parto o por morbilidad del embarazo presentan una prevalencia de serología positiva para SARS-CoV-2 del 5.28\%; mayormente son asintomáticas. No se descarta posibles efectos del virus SARS-CoV-2 
ante los hallazgos de parto pretermino, ruptura prematura de membrana y aborto espontaneo. Aunque estos datos son limitados, permiten orientar investigaciones y mejorar nuestras estrategias preventivas durante el embarazo en el contexto de la actual pandemia por COVID-19.

Financiamiento: autofinanciado.

Conflicto de intereses: Los autores declaran no tener ningún conflicto de intereses.

\section{REFERENCIAS BIBLIOGRÁFICAS}

1. OMS. Nuevo coronavirus - China. 12.01.2020. https://www. who.int/csr/don/12-january-2020-novel-coronavirus-china/es/

2. Xu X, Chen P, Wang J, et al. Evolution of the novel coronavirus from the ongoing Wuhan outbreak and modeling of its spike protein for risk of human transmission. Sci China Life Sci 2020; 63: 457-460.

3. WHO. Coronavirus disease (COVID-19) outbreak. 2020. (accessed Ene 31, 2020). https://www.who.int/emergencies/diseases/novel-coronavirus-2019/situation-reports

4. WHO. Coronavirus disease (COVID-19) outbreak. 2020. (accessed Jun 4, 2020). https://www.who.int/emergencies/diseases/novel-coronavirus-2019/situation-reports

5. Análisis Epidemiológico de la Situación Actual de COVID-19 en el Perú, basado en la información de la Vigilancia Epidemiológica y la Investigación de Campo. Centro Nacional de Epidemiologia, Prevención y Control de Enfermedades. 05.05.2020 https://www.dge.gob.pe/portal/docs/notas_prensa/2020/notaprensa032020.pdf

6. MINSA: Casos confirmados por Coronavirus Covid-19 ascienden a 183198 en el Perú (Comunicado N 121). https:// www.gob.pe/institucion/minsa/noticias/177267-minsa-casos-confirmados-por-coronavirus-covid-19-ascienden-a-183-198-en-el-peru-comunicado-n-121

7. Zeng $\mathrm{H}, \mathrm{Xu} \mathrm{C}$, Fan J, et al. Antibodies in infants born to mothers with COVID-19 pneumonia. JAMA. 2020. doi: 10.1001/ jama.2020.4861 [Epub ahead of print]

8. Lan Dong, Jinhua Tian, et al. Possible Vertical Transmission of SARS-CoV-2 From an Infected Mother to Her Newborn. JAMA, March 26, 2020. doi:10.1001/jama.2020.4621
9. Dashraath P, Jing Lin Jeslyn W, Mei Xian Karen L, Li Min L, Sarah L, Biswas A, Arjandas Choolani M, Mattar C, Lin SL, Coronavirus Disease 2019 (COVID-19) Pandemic and Pregnancy, American Journal of Obstetrics and Gynecology (2020), doi: https://doi.org/10.1016/j.ajog.2020.03.021.

10. Hegewald MJ, Crapo RO. Respiratory Physiology in Pregnancy. Clin Chest Med. 1 de marzo de 2011;32(1):1-13.

11. Instituto Nacional Materno Perinatal. Oficina de Estadistica e Informatica. Boletin Estadistico 2018 [Internet]. Lima - Perú: Instituto Nacional Materno Perinatal; 2019 [citado 13 de mayo de 2020]. Disponible en: https://www.inmp.gob.pe/institucional/boletines-estadisticos/1422371837

12. Breslin N, Baptiste C, Gyamfi-Bannerman C, Miller R, Martinez R, Bernstein K, et al. COVID-19 infection among asymptomatic and symptomatic pregnant women: Two weeks of confirmed presentations to an affiliated pair of New York City hospitals. Am J Obstet Gynecol MFM. 9 de abril de 2020;100118.

13. Crovetto F, Crispi F, Llurba E, Figueras F, Gómez-Roig MD, Gratacós E. Seroprevalence and presentation of SARSCoV-2 in pregnancy. The Lancet [Internet]. 6 de agosto de 2020 [citado 10 de agosto de 2020];0(0). Disponible en: https://www.thelancet.com/journals/lancet/article/PIIS01406736(20)31714-1/abstract

14. Knight M, Bunch K, Vousden N, Morris E, Simpson N, Gale $\mathrm{C}$, et al. Characteristics and outcomes of pregnant women admitted to hospital with confirmed SARS-CoV-2 infection in UK: national population based cohort study. BMJ [Internet]. 8 de junio de 2020 [citado 10 de agosto de 2020];369. Disponible en: https://www.bmj.com/content/369/bmj.m2107

15. Di Mascio D, Khalil A, Saccone G, Rizzo G, Buca D, Liberati $\mathrm{M}$, et al. Outcome of coronavirus spectrum infections (SARS, MERS, COVID-19) during pregnancy: a systematic review and meta-analysis. Am J Obstet Gynecol MFM. 1 de mayo de 2020;2(2, Supplement):100107.

Correspondencia:

Marcos Espinola Sánchez.

Dirección: Jr. Santa Rosa, 941. Cercado de Lima, Lima, Perú.

Teléfono: 913814091 